\title{
Acquired Cisplatin Resistance in Human Ovarian Cancer Cells Is Associated with Enhanced Repair of Cisplatin-DNA Lesions and Reduced Drug Accumulation
}

\author{
Ricardo J. Parker, ${ }^{\star}$ Alan Eastman, ${ }^{\ddagger}$ Frieda Bostick-Bruton," and Eddie Reed ${ }^{\star}$ \\ ${ }^{*}$ Medicine Branch, National Cancer Institute, Bethesda, Maryland 20892; and ${ }^{\ddagger}$ Department of Pharmacology \\ and Toxicology, Dartmouth Medical School, Hanover, New Hampshire 03755
}

\begin{abstract}
Studies were undertaken to investigate acquired resistance to cisplatin in human ovarian cancer cells. The cell lines $\mathbf{A 2 7 8 0}$ and $A 2780 / C P 70$ were studied to assess their respective characteristics of drug accumulation and efflux, cytosolic inactivation of drug, and DNA repair. All experiments were performed using 1-h drug exposures. The A2780/CP70 cell line was 13fold more resistant to cisplatin than $\mathbf{A} 2780$ cells. When studied at their respective IC50 doses, drug accumulation rates were similar for the two cell lines. However, the resistant cell line was twofold more efficient at effluxing drug, which was associated with reduced total drug accumulation for equivalent micromolar drug exposures. At equivalent levels of total cellular drug accumulation, the two cell lines formed the same levels of cisplatin-DNA damage, suggesting that cytosolic inactivation of drug does not contribute to the differential in resistance between these cell lines. Resistant cells were also twofold more efficient at repairing cisplatin-DNA lesions in cellular DNA and in transfected plasmid DNA. We conclude that in these paired cell lines, alterations in drug uptake/efflux and in DNA repair are the major contributing factors to acquired resistance to cisplatin. (J. Clin. Invest. 1991. 87:772-777.) Key words: cisplatin - ovarian cancer - resistance - DNA repair • atomic absorption spectrometry • chloramphenicol acetyltransferase • pRSVcat
\end{abstract}

\section{Introduction}

The cytotoxicity of cis-diamminedichloroplatinum(II) (cisplatin) against cells in culture has been found to be directly related to total platinum binding to DNA (1), to cisplatin-DNA interstrand cross-links (2), and to the formation of intrastrand bidentate diammineplatinum-DNA adducts at the $\mathrm{N} 7$ positions of the $d(G p G)$ and $d(A p G)$ sites in DNA $(3,4)$. Consistent with the above observations, recent studies suggest that DNA repair capacity for one or more of these lesions may be a major determinant of resistance to cisplatin in L1210 cells (5), human ovarian cancer cells (6), human testicular cancer cells, and human bladder cancer cells (7).

Address correspondence to Dr. E. Reed, Medicine Branch/Clinical Pharmacology Branch, National Cancer Institute, National Institutes of Health, 9000 Rockville Pike, Bldg. 10, Rm. 6N119, Bethesda, MD 20892.

Received for publication 1 June 1990 and in revised form 19 September 1990

J. Clin. Invest.

(c) The American Society for Clinical Investigation, Inc.

0021-9738/91/03/0772/06 \$2.00

Volume 87, March 1991, 772-777
Studies of the relationship of DNA repair to cisplatin resistance in mammalian cells are being conducted by several groups. The most extensive studies done to date are those of Eastman and colleagues in L1210 cells in which varying levels of acquired resistance to cisplatin has been developed $(5,8)$. In those studies, HPLC techniques were used to show that resistant sublines were much more effective at removing cisplatinDNA lesions from cellular DNA as compared with the more sensitive sublines. However, the extent of repair did not correlate completely with the extent of resistance to cisplatin, suggesting that enhanced DNA repair was one of several determinants of the level of resistance in L1210 cells. Using immunochemical methodology, Bedford and colleagues reported that cisplatin-resistant human bladder cancer cell lines have greater capacity to remove intrastrand adducts from cellular DNA than relatively sensitive testicular cancer cell lines (7). It is unclear whether these antibody techniques measure DNA lesions that are unique to the antibody, or are consistent with other techniques such as HPLC or atomic absorption spectrometry $(\mathrm{AAS})^{1}(9)$. Immunochemical methodology has been used by Reed and colleagues to investigate drug binding to DNA and its relationship to cell kill after therapeutic levels of drug exposure in human ovarian cancer cells in vitro (10). When sensitive and resistant sublines of A2780 were studied, cells which were more resistant to cisplatin required more drug binding to DNA to effect the same level of cell kill. Maduda and colleagues have shown that total cisplatin-DNA damage is related to cytotoxicity in human ovarian cancer cells and that aphidicholin can inhibit the repair of that damage and thereby increase cell kill (11). Using another assay for DNA repair, Lai et al. have also reported that in human ovarian cancer cells, increased levels of unscheduled DNA synthesis is associated with resistance to cisplatin (6).

Although these studies suggest that DNA repair may be important in platinum drug resistance in human ovarian cancer, no studies have been published to date which directly address the relative contributions of drug uptake and repair of DNA damage in human ovarian cancer cells. We therefore investigated drug uptake and DNA repair of cisplatin-DNA lesions in the human ovarian cancer cells A2780 and A2780/CP70. These experiments involved measurements of sensitivity to cisplatin, AAS measurements of total drug accumulation and efflux by these cells, AAS measurements of adduct formation and removal from cellular DNA, and the rela-

1. Abbreviations used in this paper: AAS, atomic absorption spectrometry; CAT, chloramphenicol acetyltransferase; $d(A p G)$, adjacent adenine and guanine moieties in an intact DNA strand; $d(\mathrm{GpG})$, adjacent guanine moieties in an intact DNA strand; IC50, dose of drug that reduces survival rate in a cell line to $50 \%$ of the control value; IC 90 , dose of drug that reduces survival rate in a cell line to $10 \%$ of the control value ( $90 \%$ cell kill); pRSVcat, designation given to the plasmid used in these studies by its developers (see reference 16). 
tive abilities of these cell lines to reactivate cisplatin-damaged plasmid DNA.

\section{Methods}

Cell culture. The ovarian cancer cell lines A2780 and A2780/CP70 were generously provided by Dr. Robert Ozols. The L1210/0 cell line has been previously characterized $(5,8)$. Ovarian cancer cells were cultured in monolayer using RPMI 1640 media, supplemented with $10 \%$ FCS, $0.2 \mathrm{U} / \mathrm{ml}$ of human insulin, and penicillin/streptomycin (Gibco, Grand Island, NY). Cells were grown in a humidified $5 \% \mathrm{CO}_{2}$ mixture with ambient air at $37^{\circ} \mathrm{C}$. L1210 cells were grown in suspension under similar conditions, but with the absence of insulin in the medium.

Sensitivity to cisplatin in ovarian cancer cells was assessed in colony growth assays using six well plates, each well $35 \mathrm{~mm}$ in diameter. Cells were plated at 500 cells per well and cisplatin treatments were performed as $1-\mathrm{h}$ drug exposures on the day after plating. Cisplatin was initially dissolved in sterile phosphate buffered saline at $1 \mathrm{mg} / \mathrm{ml}$, and dilutions from this $1 \mathrm{mg} / \mathrm{ml}$ solution were made into RPMI media to obtain the desired drug treatment concentration. Cells were allowed to grow for a period of 7-10 d, at which time colonies were stained with a methylene blue solution of $0.167 \mathrm{~g} \%$ in absolute methanol. Visible colonies were counted by hand. Drug treatments were done in triplicate at each dose in each individual experiment. The value obtained in wells where no drug was added was assigned the value of $100 \%$ growth.

Measurement of cellular drug accumulation rate and efflux. A2780 and A2780/CP70 cell lines were treated at their respective IC50 cisplatin doses for the purpose of measuring cellular drug accumulation and efflux. Cells were exposed to cisplatin for $1 \mathrm{~h}$ in all experiments. To measure drug accumulation, we harvested cells at $30 \mathrm{~min}$ and $1 \mathrm{~h}$. To measure drug efflux, cells were treated for $1 \mathrm{~h}$ and harvested at the following time points: 30 min after drug removal, $1 \mathrm{~h}, 2 \mathrm{~h}$, and $4 \mathrm{~h}$. Total cellular drug accumulation and efflux in these two cell lines were compared by assigning the value of $100 \%$ to that drug level achieved at $1 \mathrm{~h}$, and assessing all other values relative to the $100 \%$ value.

Measurement of platinum in cellular DNA and cellular drug accumulation. In one set of experiments, we sought to establish the relationship between cisplatin dose and cisplatin-DNA adduct formation in the sensitive and resistant cell lines. Both cell lines were treated in monolayer with cisplatin doses of $0,10,20,40$, or $60 \mu \mathrm{M}$ cisplatin for $1 \mathrm{~h}$; cells were immediately harvested; DNA was isolated (12) and measured by absorbance at $260 \mathrm{~nm}$; and total platinum per unit DNA was measured by AAS (13). In a separate set of experiments both cell lines were treated under the same conditions for the purpose of measuring cellular accumulation of drug. After a 1-h exposure, cells were immediately harvested and frozen at $-20^{\circ} \mathrm{C}$. The cell pellets were later "wetashed" according to the method of McGahan (14), and total cellular drug accumulation was measured as femtomoles of platinum per $10^{7}$ cells.

To assess the ability of these cells to remove platinum from cellular DNA, cells were plated in T-150 flasks and allowed to grow in log phase with changes of fresh media twice weekly. Cells were labeled with ${ }^{3} \mathrm{H}$ thymidine, using media with a ${ }^{3} \mathrm{H}$-thymidine concentration of $0.1 \mu \mathrm{Ci}$ / $\mathrm{ml}$. Cells were labeled with ${ }^{3} \mathrm{H}$ overnight, after which fresh media was placed onto the cells and incubation was again carried out overnight. At this time, cells were exposed to various cisplatin drug concentrations for $1 \mathrm{~h}$. In designated experiments, cells were treated at their respective IC50s, as was assessed by colony formation (see above). In other experiments, the A2780 cell line was incubated with $22 \mu \mathrm{M}$ drug to obtain a level of DNA platination equivalent to that obtained in the A2780/CP70 cell line after its IC50 exposure of $40 \mu \mathrm{M}$. The $22-\mu \mathrm{M}$ drug dose was based on extrapolation from the linear regression equation for A2780 cells from Fig. 3 (see below). After ${ }^{3} \mathrm{H}$ labeling, an aliquot of cells was harvested before drug treatment (time zero). After a 1-h drug exposure, cells were harvested at the following time points: $1 \mathrm{~h}$ (immediately at the end of the cisplatin exposure), 3, 5, 7, and $24 \mathrm{~h}$. Cells were frozen immediately at $-20^{\circ} \mathrm{C}$.
DNA was isolated on cesium chloride density gradients, yielding DNA which was $99.6 \%$ free of contamination (12). This DNA was dialyzed against four exchanges of double distilled water over $\sim 36 \mathrm{~h}$. DNA was then measured by absorbance at $260 \mathrm{~nm}$. ${ }^{3} \mathrm{H}$-Thymidine content was assessed by liquid scintillation counting and platinum content was assessed by AAS (13). A decrease in the specific radioactivity of DNA (disintegrations per minute per microgram DNA) at each time point compared to that obtained at $1 \mathrm{~h}$ represents the amount of replication that occurred. This ratio was used to correct the values of platinum content of cellular DNA.

Reactivation studies of cisplatin-damaged pRSVcat. The JM-109 strain of $E$. coli, containing the plasmid pRSVcat, was generously provided by the laboratory of Dr. Bruce Howard of the National Cancer Institute. Plasmid was grown and purified using standard growth procedures and the alkali lysis method as described by Maniatis (15). Purified pRSVcat was platinated according to the method of Eastman in $0.02 \mathrm{M}$ sodium perchlorate, $\mathrm{pH} 5.5$ (16). The level of plasmid platination was confirmed by AAS. Plasmid was transfected into ovarian cancer cells using a modification of the DEAE-dextran method (17). Immediately after trypsinization, transfection was carried out with cells in suspension, using 3-5 $\times 10^{6}$ cells in $1 \mathrm{ml}$ of media with a DEAE-dextran concentration of $250 \mu \mathrm{g} / \mathrm{ml}$ and a defined quantity of plasmid for $3 \mathrm{~h}$. After completion of the transfection exposure, cells were pelleted, washed, resuspended in fresh media, and plated in monolayer. Cells were harvested at $48 \mathrm{~h}$ and CAT activity was measured according to the method of Neumann (18) as modified by Eastman (19). The level of chloramphenicol acetyltransferase (CAT) activity associated with zero level of cisplatin molecules per plasmid was assigned a value of $100 \%$ CAT activity and the CAT activity associated with damaged plasmid was assessed relative to this control value.

Measurement of efficiency of pRSVcat transfection. The effect of different levels of platinum modification on the transfection efficiency of the pRSVcat plasmid was assessed using a modification of the transfection method described above. Plasmid was prepared in a standard fashion with the exception of the addition of ${ }^{3} \mathrm{H}$-thymidine to the $E$. coli culture medium at a concentration of $0.1 \mu \mathrm{Ci} / \mathrm{ml} .{ }^{3} \mathrm{H}$-labeled pRSVcat was harvested as described above and the specific activity of the ${ }^{3} \mathrm{H}$ relative to plasmid DNA was assessed. ${ }^{3} \mathrm{H}$-labeled $\mathrm{pRSV}$ cat was then platinated as outlined above. Cisplatin-modified, ${ }^{3} \mathrm{H}$-labeled, pRSVcat plasmid was then transfected into the respective cell lines. 50 $\mu \mathrm{g}$ of plasmid DNA and 3-6 $\times 10^{6}$ cells were used for each transfection. After the 3-h transfection period cells were washed, exposed to $1 \mu \mathrm{g} / \mathrm{ml}$ of DNase for $15 \mathrm{~min}$ at $37^{\circ} \mathrm{C}$ to remove extracellular DNA, and then washed again. The amount of ${ }^{3} \mathrm{H}$ activity transfected into cells with plasmid that contained no platinum was assigned $100 \%$ transfection activity, and plasmid with increasing levels of platination was assessed relative to this control value.

\section{Results}

Differences in survival between cell lines. The relative sensitivity of the two cell lines to cisplatin as assessed by colony formation is shown in Fig. 1. The IC50 of cisplatin, based on a 1-h drug exposure, is $3 \mu \mathrm{M}$ in the A2780 cell line; the IC90 is reached at $30 \mu \mathrm{M}$. By contrast, in the A2780/CP70 cell line, the IC50 is reached at $40 \mu \mathrm{M}$ and the IC90 is reached at $100 \mu \mathrm{M}$. The difference between the cell lines in the IC50 drug dose demonstrates a 13-fold difference in cellular sensitivity to cisplatin for these two cell lines.

Measurement of cellular drug accumulation and efflux. We measured the relative rates of cellular accumulation and efflux of cisplatin, during and after 1-h exposures to IC50 drug doses in the two cell lines. Shown in Fig. 2 are data for A2780 (IC50 $=3 \mu \mathrm{M})$ and A2780/CP70 (IC50 $=40 \mu \mathrm{M})$ with each data point representing the mean of four determinations. When assessed as a percentage of maximal drug accumulation, the two 


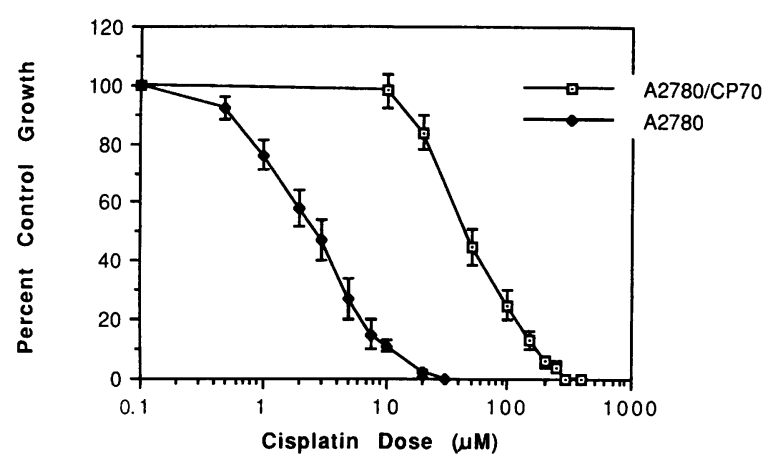

Figure 1. Sensitivity to cisplatin was assessed by colony formation assay in A2780, wild type cells (solid diamonds) and A2780/CP70 cells (open squares). Cells were plated at 500 cells per well; drug exposures were for $1 \mathrm{~h}$ in triplicate wells; cells were grown for 7-10 d; visible colonies were counted by hand; and values were expressed relative to those wells where no drug was used (100\% control value). Each data point is the mean and standard deviation from four separate determinations.

\section{A}

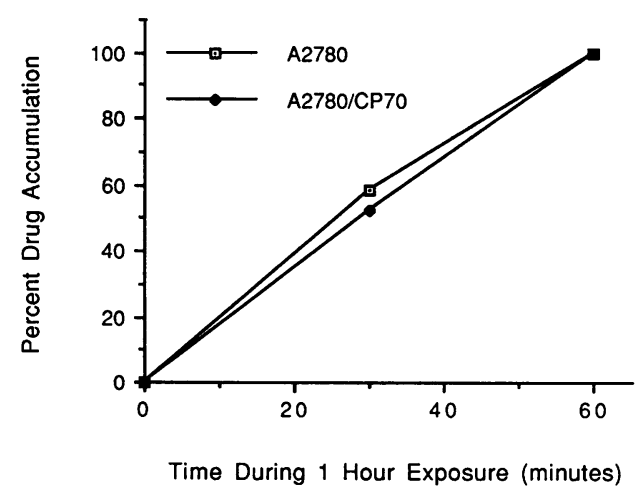

B

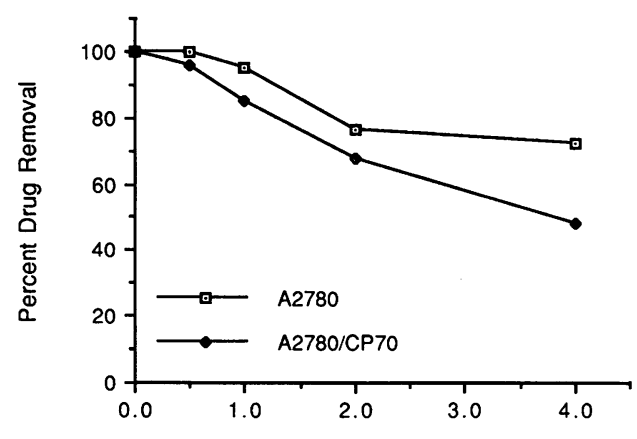

Time After 1 Hour Exposure (hours)

Figure 2. Cellular drug accumulation $(A)$ and efflux $(B)$ were determined in A2780 (open squares) and A2780/CP70 (solid diamonds) during and after IC50 drug exposures for $1 \mathrm{~h}$. The IC50s were: A2780, $3 \mu \mathrm{M}$; A2780/CP70, $40 \mu \mathrm{M}$. Cells were treated as described in the text. Each data point is the mean of four separate determinations. Absolute values for drug uptake in the respective cell lines are graphically shown in Fig. 4. cell lines accumulated drug at the same rate over the 1-h time period. At $30 \mathrm{~min}$ of the $1 \mathrm{~h}$ exposure, A2780 accumulated $58.5 \%$ of total cellular platinum and A2780/CP70 accumulated $52.5 \%$ (Fig. $2 \mathrm{~A}$ ).

We then measured drug efflux over $4 \mathrm{~h}$, after a 1-h treatment with IC50 drug doses (Fig. 2 B). For A2780, cellular platinum levels changed gradually during the observation period, and at the end of $4 \mathrm{~h}$ a total of $27.4 \%$ of accumulated cellular platinum had been removed from these cells. Cisplatin-resistant A2780/CP70 cells demonstrated enhanced efflux as compared to A2780 cells. After 4 h, $51.4 \%$ of accumulated cellular platinum had been removed.

Measurement of drug-DNA binding after defined drug exposures. We sought to establish the level of cisplatin-DNA adduct formation in the two cell lines after equivalent cisplatin drug exposures (Fig. 3). Each data point is the mean and standard deviation of four determinations. In the sensitive cell line, the relation between drug dose and adduct level approaches linearity up to $40 \mu \mathrm{M}$, and then appears to plateau. Linear regression analysis of these data up to the cisplatin dose of $40 \mu \mathrm{M}$ yields the equation $y=1.2 x+2.6, r=0.98$, and through the dose of $60 \mu \mathrm{M}$, the linear regression equation is $y=0.96 x+0.74, r$ $=0.94$. In the resistant cell line the relation also approaches linearity, and the equation of the linear regression analysis is $y$ $=0.55 x+0.77, r=0.97$. Based on the slopes of the linear portions of the curves, approximately twofold more cisplatin was needed in the resistant cells to attain the same level of DNA damage. These values also demonstrate that $1 \mathrm{Pt} / 10^{6}$ nucleotides produces an IC50 in A2780 cells while $1 \mathrm{Pt} / 1.4 \times 10^{5}$ nucleotides produces a comparable toxicity in A2780/CP70 cells. The former value is consistent with the published value for $\operatorname{L} 1210$ cells at their $\operatorname{IC} 50(5,8)$.

Assessment of total cellular accumulation of drug. We next measured cellular accumulation of cisplatin under the conditions used to measure drug-DNA binding as discussed above. Fig. 4 shows these data with each data point representing the mean and standard deviation of four determinations. As was the case with respect to DNA binding, the relationship between drug dose and total cellular accumulation of drug approached linearity for both cell lines. In the sensitive cell line, the linear regression equation is $y=0.20 x+0.60, r=0.98$; and in the

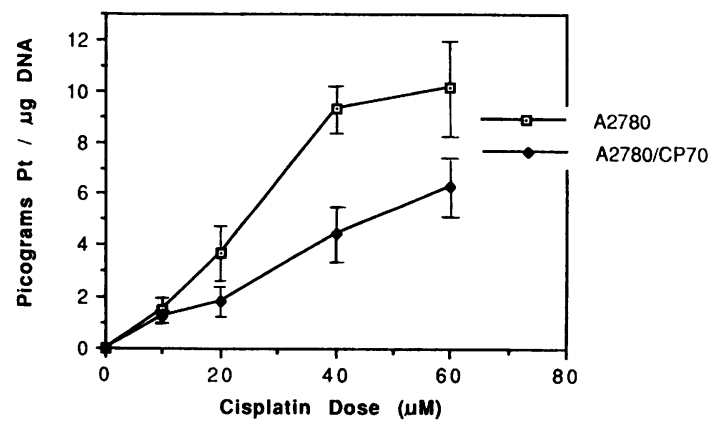

Figure 3. Total DNA bound platinum was measured in A2780, wild type cells (open squares) and A2780/CP70 cells (solid diamonds) after defined drug exposures for $1 \mathrm{~h}$. Cells were harvested immediately after drug exposure. DNA was isolated by cesium chloride bouyant density gradient centrifugation, and total platinum content was measured using atomic absorption spectroscopy with Zeeman background correction. Each data point is the mean and standard deviation from four separate determinations. 


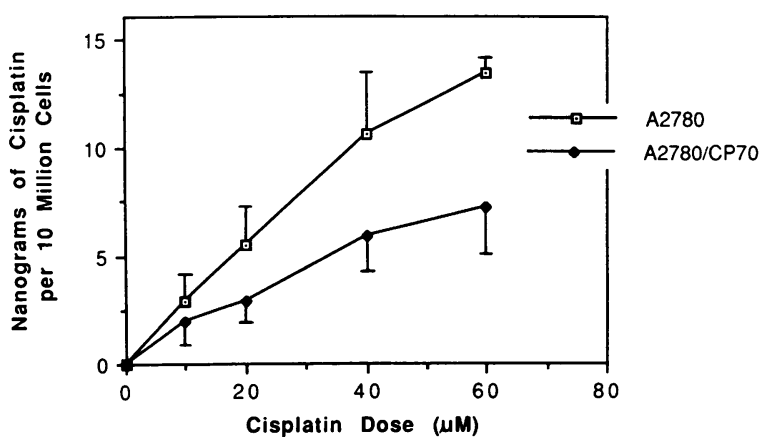

Figure 4. Total cellular accumulation of drug was measured in A2780, wild type cells (open squares) and A2780/CP70 cells (solid diamonds) after defined drug exposures for $1 \mathrm{~h}$. Cells were washed with PBS, trypsinized, and "wet-ashed" as described in the text. Each data point is the mean and standard deviation from four separate determinations.

resistant cell line the equation is $y=0.10 x+0.50, r=0.97$. Based on the slopes of the linear portions of the curves, about twofold more drug is needed in the resistant cells to attain the same level of cellular drug accumulation. When data on cellular accumulation are directly compared with DNA adduct level, the two cell lines form curves that are virtually superimposable (Fig. 5). Thus, the level of drug uptake is the primary determinant of DNA adduct level in these two cell lines and cytosolic drug inactivation appears not to be a factor in determining any difference between cell lines in the amount of DNA-bound drug. For example, both cellular accumulation of drug and DNA platination are equivalent in the two cell lines at the respective cisplatin doses of $20 \mu \mathrm{M}$ in the $\mathrm{A} 2780$ cells, and $40 \mu \mathrm{M}$ in A2780/CP70 cells.

Removal of platinum from cellular DNA. For the purpose of comparing the two cell lines in their respective ability to remove platinum from cellular DNA, two approaches were taken. In the first, an IC50, 1-h dose was chosen for treating the respective cell lines, and we followed platinum removal from cellular DNA with time. In the second approach, we compared the sensitive cell line with the resistant cell line when both were "loaded" with the same level of DNA bound cisplatin (Fig. 6).

After an IC50 dose in the A2780 cell line, the peak platinum level seen after exposure was $1.0 \mathrm{pg} / \mu \mathrm{g}$ DNA (Fig. 6). This was followed by a 6 -h phase of rapid removal from cellular DNA to a level of $0.5 \mathrm{pg} / \mu \mathrm{g}$ DNA. This was followed by a plateau, indicating no further removal of platinum during the remaining time of the 24 -h observation period. For the

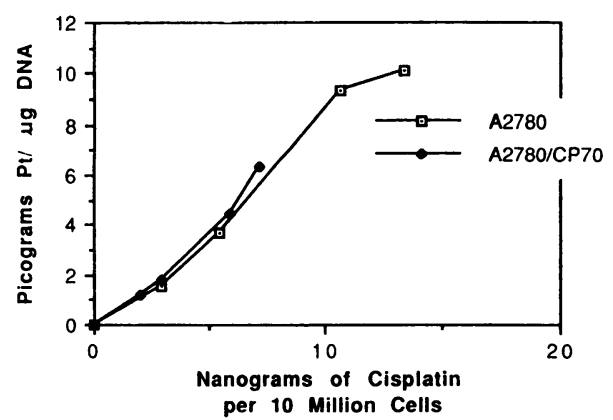

Figure 5. Data shown in Figs. 3 and 4 are transposed to give plots of drug accumulation versus DNA adduct level in the two ovarian cancer cell lines. As shown, the curves are virtually superimposable.

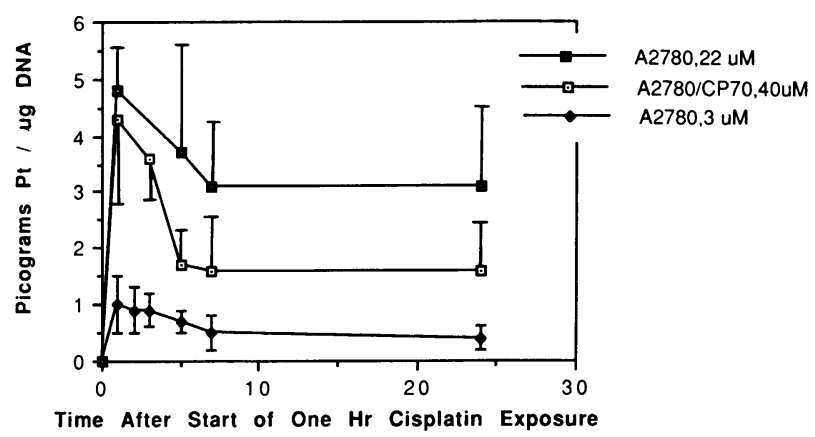

Figure 6. The removal of elemental platinum from cellular DNA with time was assessed in the two cell lines in three different sets of experiments. In one set, A2780, wild type cells were treated at their IC50 dose of $3 \mu \mathrm{M}$ for $1 \mathrm{~h}$ and total platinum per unit DNA was measured at defined time points after the beginning of the cisplatin exposure (solid diamonds). In a second set of experiments, A2780/ CP70 cells were treated at their IC50 dose of $40 \mu \mathrm{M}$ for $1 \mathrm{~h}$ and total platinum per unit DNA was followed (open squares). In a third set, A2780, wild type cells were treated with $22 \mu \mathrm{M}$ cisplatin for $1 \mathrm{~h}$ so as to attain a level of DNA modification equivalent to that seen in the resistant cell line after its $40 \mu \mathrm{M}$ exposure (closed squares). Each data point is the mean and standard deviation from three or from four separate determinations.

A2780/CP70 cell line, after an IC50 dose which is 13 -fold higher than the A2780 IC50 dose, the peak platinum level was $4.3 \mathrm{pg} / \mu \mathrm{g}$ DNA (Fig. 6). A phase of rapid removal occurred over the next $4 \mathrm{~h}$, down to a level of $1.6 \mathrm{pg}$ platinum per $\mu \mathrm{g}$ DNA. This was followed by a plateau phase during the remainder of the 24-h period of observation. Therefore, in terms of the absolute quantity of platinum, the peak levels seen after an IC50 exposure is fourfold higher in the resistant cell line than in the sensitive cell line.

When viewed as a percentage of the maximal value of platinum bound to DNA, the resistant cell line removed $63 \%$ of its total platinum load from cellular DNA in the first $6 \mathrm{~h}$, whereas the sensitive cell line was capable of removing only $50 \%$. In both cases, this was after IC50 drug exposures. When assessed as absolute quantity of adduct, the amount of platinum removed from cellular DNA during the observation period is fivefold greater in the resistant cell line. In both cell lines, some portion of the adduct load persists for at least $24 \mathrm{~h}$ after the cisplatin exposure. The amount of adduct that persists is threefold greater in the resistant cell line as compared to the sensitive cell line ( 1.6 vs. $0.5 \mathrm{pg} / \mu \mathrm{g}$ DNA).

Also in Fig. 6 are data from the sensitive cell line when incubated at a cisplatin dose of $22 \mu \mathrm{M}$. At this dose, the adduct level at $1 \mathrm{~h}$ was $4.8 \mathrm{pg} / \mu \mathrm{g}$ DNA, which approximates the adduct level seen in the resistant cell line when treated at its IC50 of 40 $\mu \mathrm{M}$. Over the next $6 \mathrm{~h}$, the adduct level was reduced to 3.1 $\mathrm{pg} / \mu \mathrm{g}$ DNA: i.e., $35 \%$ of the total platinum load was removed. This was followed by a plateau, as was seen in the other two cases. As shown, the sensitive cell line is less able to remove cisplatin from its cellular DNA when loaded to this level of platination (35\% removal over $6 \mathrm{~h}$, vs. $63 \%$ removal in the resistant cell line).

Efficiency of transfection of pRSVcat. Studies were performed in the two cell lines to assess the influence of plasmid platination on the relative efficiency of transfection of pRSVcat. Levels of plasmid platination studied included 0,5 , 10,20 , and 40 platinum molecules per plasmid. In these exper- 
iments, we assessed the relative efficiency of transfection as that percentage of plasmid that was associated with the cell after DNase treatment. In both the A2780 and the A2780/CP70 cell lines, the total percentage of control plasmid that was associated with the cells after DNase treatment was $<3 \%$ (data not shown). In each experiment, the level of plasmid platination did not substantively alter the amount of plasmid that was transfected into cells (Fig. 7).

Different activities of host cell reactivation of $p R S V$ cat. To compare the cell lines' abilities to reactivate the pRSVcat plasmid, transfections were performed in three cell lines using a single batch of pRSVcat. The A2780 and A2780/CP70 cell lines were studied, along with the L1210/0 cell line which served as an internal control in these experiments because it is known to perform virtually no repair of cisplatin-DNA lesions $(8,16)$. Data from these cell lines are shown in Fig. 8 . In the L1210/0 cell line, host cell reactivation of pRSVcat was totally consistent with what has been previously reported, with a $\mathrm{B}_{0}$ (amount of DNA damage constituting one lethal hit to transfected plasmid DNA) of five platinum molecules per plasmid $(8,16)$. Levels of CAT activity equivalent to $100 \%$ of control were seen in the A2780 cell line up to a modification level of eight platinum molecules per plasmid. In the A2780/CP70 cell line, $100 \%$ of control activity was seen at modification levels up to 16 platinum molecules per plasmid. Levels of CAT activity $<10 \%$ of control were seen at 32 platinum molecules per plasmid in the sensitive cell line, and 56 molecules per plasmid in the resistant cell line. When these data are represented graphically, the $\mathrm{B}_{0}$ for the two cell lines ( $37 \%$ control activity) differ by a factor of approximately two: 16 platinum molecules per plasmid in the sensitive cell line, and 27 molecules per plasmid in the resistant cell line.

\section{Discussion}

The A2780/CP70 cell line exhibits acquired resistance to cisplatin which resulted from continuous stepwise exposure to cisplatin over many months (6), and was derived from the relatively cisplatin-sensitive A2780 cell line. The resistant cell line shows a twofold reduced drug accumulation, enhanced drug efflux, and twofold reduced DNA platination. For a given level of drug accumulation, the level of DNA damage was the same,

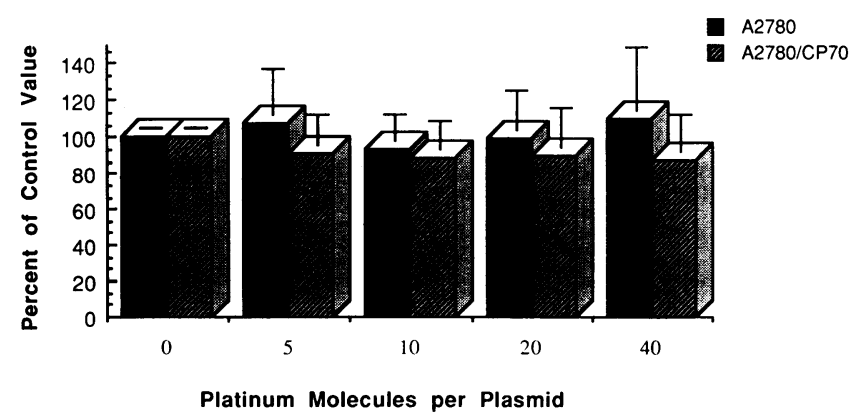

Figure 7. The effect of cisplatin modification on the transfection efficiency of pRSVcat into A2780, wild type cells (solid bars) or A2780/CP70 cells (hatched bars) was assessed by preparing ${ }^{3} \mathrm{H}$-labeled plasmid, modifying that plasmid with cisplatin, and then transfection into the respective cell line. After transfection, cells were DNase treated and immediately placed into scintillation fluid for assessment of cellular ${ }^{3} \mathrm{H}$ content. Each bar represents the mean and standard deviation from four separate determinations.

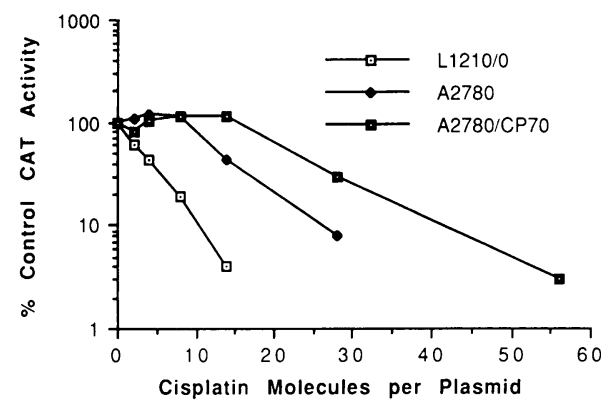

Figure 8. Host cell reactivation of cisplatin-damaged pRSVcat was assessed as the percent reduction from control levels of CAT activity, as a function of the level of cisplatin modification. Transfection was carried out with cells in suspension, using 3-5 $310^{6}$ cells in $1 \mathrm{ml}$ of media with a DEAE-dextran concentration of $250 \mu \mathrm{g} / \mathrm{ml} .10 \mu \mathrm{g}$ of plasmid was used for each transfection. Each data point is the mean of two to three determinations.

regardless of the cell line. Increased levels of cytosolic sulfhydryl-containing proteins has been described as inducing cisplatin resistance in human ovarian cancer cells. Whereas published evidence supports the contention that drug inactivation in the cytosol by metallothionein (20) and/or glutathione (21) may contribute to cisplatin resistance, our data suggests that in these cells, this mechanism does not contribute to the differential in resistance. The drug-resistant cells also show an increased ability to remove platinum from cellular DNA as compared to the sensitive cell line, and show an augmented ability to reactivate the $\mathrm{pRSV}$ cat plasmid after cisplatin-induced DNA damage. These independent measures of DNA repair capability are both enhanced in the cisplatin-resistant cell line by a factor of two.

To assess platinum removal from cellular DNA, these cell lines were compared at their respective IC50 doses so as to obtain measures of how these cells handle biologically relevant levels of drug-induced DNA damage. The IC50 doses were chosen because they represent at least one measure of biologically equivalent drug doses. After the 1-h cisplatin exposure, at least fourfold more cisplatin-DNA damage is necessary in the resistant cell line to attain the same level of cell kill as in the sensitive line. We obtained these measurements using atomic absorption spectrometry which measures total DNA bound drug after cisplatin exposures (13). Atomic absorption spectrometry is possibly a more reliable assessment of total DNA bound drug than other methods of adduct measurement thus far reported (22). However, there is debate as to whether total DNA bound drug is of primary importance, or if one or more of the defined specific platinum-DNA lesions is of primary importance in inducing cell kill (22).

The ability to "reactivate" UV-damaged or chemically damaged plasmid or viral genomes that have been introduced into cells is related to DNA repair capability. This has been shown for various cell lines using a number of strains of adenovirus (23-25) and using UV-damaged or cisplatin-damaged pRSVcat $(8,16,26)$. In this study, the relative abilities of these cell lines to reactivate cisplatin-damaged $\mathrm{pRSV}$ cat parallels the cell lines' abilities to remove platinum from cellular DNA, when these cells are DNA-modified to the same level with cisplatin. This suggests that host cell reactivation in this setting may serve as an indication of a cell line's relative ability to perform repair of cisplatin-DNA lesions. 
Direct and indirect evidence suggests that DNA repair may also be important in human ovarian cancer in vivo. CisplatinDNA adduct persistence is directly contributory to measured adduct level in human leukocytes (27), which in turn is directly related to disease response (27-29). Data presented in this report demonstrate that adduct persistence at $24 \mathrm{~h}$ after cisplatin exposure is inversely related to DNA repair capability. In UV repair-deficient Chinese hamster ovary cells of complementation group I, transfection of the human DNA repair gene ERCC1 confers fivefold resistance to cisplatin and confers the ability to remove cisplatin from cellular DNA (30). Preliminary data show that expression of ERCC1 in fresh ovarian cancer tumor tissue is directly related to a patient's response to single-agent carboplatin chemotherapy (Dabholkar, M., and E. Reed, unpublished observations). The current report shows that a specific increase in the ability to repair cisplatin-DNA lesions is a major component, but not the only component, of acquired cisplatin resistance in human ovarian cancer cells.

In all human cell lines thus far studied, it is now apparent that cisplatin drug resistance is a multifactorial process which may exist as a combined function of drug uptake, DNA repair, and/or cytosolic drug inactivation. In established human ovarian cancer cell lines which differ biologically in that one has acquired in vitro resistance to cisplatin, the resistant cell line has reduced drug accumulation that correlates with reduced DNA platination. This suggests that cytosolic drug inactivation is not a contributor to the differential in resistance in these cells, and reduced DNA platination only accounts for twofold resistance. The other identified contributor to resistance is the enhanced DNA repair capability for cisplatin-DNA damage, as assessed by removal of total platinum from cellular DNA and the enhanced ability to repair cisplatin-damaged pRSVcat.

\section{References}

1. Knox, R. J., F. Friedlos, D. A. Lydall, and J. J. Roberts. 1986. Mechanism of cytotoxicity to anticancer platinum drugs: evidence that cis-diamminedichloroplatinum(II) and cis-diammine-(1,1-cyclobutanedicarboxylato)platinum(II) differ only in the kinetics of their interaction with DNA. Cancer Res. 46:19721979.

2. Zwelling, L. A., T. Anderson, and K. W. Kohn. 1979. DNA-protein and DNA interstrand cross-linking by cis- and trans-platinum(II)diamminedichloride in L1210 mouse leukemia cells and its relation to cytotoxicity. Cancer Res. 39:365-369.

3. Eastman, A. 1983. Characterization of the adducts produced in DNA by cis-diamminedichloroplatinum(II) and cis-dichloro(ethylenediamine)platinum(II). Biochemistry. 22:3927-3933.

4. Eastman, A. 1987. The formation, isolation and characterization of DNA adducts produced by the anticancer platinum complexes. Pharmacol. \& Ther. 34:155-166.

5. Eastman, A., and N. Schulte. 1988. Enhanced DNA repair as a mechanism of resistance to cis-diamminedichloroplatinum(II). Biochemistry. 27:4730-4734.

6. Lai, G., R. F. Ozols, J. F. Smyth, R. C. Young, and T. C. Hamilton. 1988. Enhanced DNA repair and resistance to cisplatin in human ovarian cancer. Biochem. Pharmacol. 37:4597-4600.

7. Bedford, P., A. M. J. Fichtinger-Schepman, S. A. Sellard, M. C. Walker, J. R. W. Masters, and B. T. Hill. 1988. Differential repair of platinum-DNA adducts in human bladder and testicular continuous cell lines. Cancer Res. 48:3019-3024.

8. Eastman, A., N. Schulte, N. Sheibani, and C. M. Sorensen. 1988. Mecha- nisms of resistance to platinum drugs. In Platinum and Other Metal Compounds in Cancer Chemotherapy. M. Nicolini, editor. Martinus Nijhoff Publishers, Boston. 178-196.

9. Poirier, M. C., M. J. Egorin, A. M. J. Fichingter-Schepman, and E. Reed. 1988. DNA adducts of cisplatin and carboplatin in tissues of human cancer patients. IARC (Int. Agency Res. Cancer) Sci. Publ. 89:313-320.

10. Reed, E., B. C. Behrens, S. H. Yuspa, M. C. Poirier, T. C. Hamilton, and R. T. Ozols. 1986. Differences in cisplatin-DNA adduct formation in sensitive and resistant sublines of human ovarian cancer cells. Proc. Am. Assoc. Cancer Res. 27:285. (Abstr.)

11. Maduda, H., T. Tanaka, H. Matsuda, and I. Kasaba. 1990. Increased removal of DNA-bound platinum in a human ovarian cancer cell line resistant to cis-diamminedichloro-platinum(II). Cancer Res. 50:1863-1866.

12. Flamm, W. G., M. L. Birnstiel, and P. M. B. Walker. 1969. Subcellular Components. Preparation and Fractionation. G. Birnie and S. M. Fox, editors. Butterworth and Co., Ltd., London. 129-155.

13. Reed, E., S. Sauerhoff, and M. C. Poirier. 1988. Quantitation of platinumDNA binding in human tissues following therapeutic levels of drug exposure. Atomic Spectroscopy. 9:93-95.

14. McGahan, M. C., and K. Tyczkowska. 1987. The determination of platinum in biological materials by electrothermal atomic absorption spectroscopy. Spectrochim. Acta. 42B:665-668.

15. Maniatis, T., E. F. Fritsch, and J. Sambrook. 1982. Molecular Cloning: A Laboratory Manual. Cold Spring Laboratory, Cold Spring Harbor, NY.

16. Sheibani, N., M. M. Jennerwein, and A. Eastman. 1989. DNA repair in cells sensitive and resistant to cis-diamminedichloroplatinum (II): host cell reactivation of damaged plasmid DNA. Biochemistry. 28:3120-3124.

17. Milman, G., and M. Herzberg. 1981. Efficient DNA transfection and rapid assay for thymidine kinase activity and viral antigenic determinants. Somatic Cell Gen. 7:161-170.

18. Neumann, J. R., C. A. Morency, and K. O. Russian. 1987. A novel assay for chloramphenicol acetyltransferase gene expression. Biotechniques. 5:444447.

19. Eastman, A. 1987. An improvement to the novel rapid assay for chloramphenicol acetyltransferase gene expression. Biotechniques. 5:730-732.

20. Andrews, P. A., M. P. Murphy, and S. B. Howell. 1987. Metallothioneinmediated cisplatin resistance in human ovarian carcinoma cells. Cancer Chemother. Pharmacol. 19:149-154.

21. Lai, G., R. F. Ozols, R. C. Young, and T. C. Hamilton. 1989. Effect of glutathione on DNA repair in cisplatin-resistant human ovarian cancer cell lines. J. Natl. Cancer Inst. 81:535-539.

22. Reed, E., and K. W. Kohn. 1990. Cisplatin and platinum analogs. In Cancer Chemotherapy: Principles and Practice. B. A. Chabner, editor. J. B. Lippincott Co., Philadelphia. 465-490.

23. Day III, R. S., D. Scudiero, and M. Dimattina. 1978. Excision repair by human fibroblasts of DNA damaged by r-7,t-8-dihydroxy-t-9,10-oxy-7,8,9,10-tetrahydrobenzo(a)pyrene. Mutat. Res. 50:383-394.

24. Day III, R. S. 1974. Cellular reactivation of ultraviolet-irradiated human adenovirus 2 in normal and xeroderma pigmentosum fibroblasts. Photochem. Photobiol. 19:9-13.

25. Day III, R. S., A. S. Guiffrida, and C. W. Dingman. 1975. Repair by human cells of adenovirus 2 damaged by psoralen plus near ultraviolet light treatment. Mutat. Res. 33:311-320.

26. Proctic-Sablijic, M., and K. H. Kraemer. 1985. One pyrimidine dimer inactivates expression of a transfected gene in xeroderma pigmentosum cells. Proc. Natl. Acad. Sci. USA. 82:6622-6626.

27. Reed, E., S. H. Yuspa, L. A. Zwelling, R. F. Ozols, and M. C. Poirier. 1986 Quantitation of cisplatin-DNA intrastrand adducts in testicular and ovarian cancer patients receiving cisplatin chemotherapy. J. Clin. Invest. 77:545-550.

28. Reed, E., R. F. Ozols, R. Tarone, S. H. Yuspa, and M. C. Poirier. 1987. Platinum-DNA adducts in leukocyte DNA correlate with disease response in ovarian cancer patients receiving platinum-based chemotherapy. Proc. Natl. Acad. Sci. USA. 84:5024-5028.

29. Reed, E., Y. Ostchega, S. Steinberg, S. H. Yuspa, R. C. Young, R. F. Ozols, and M. C. Poirier. 1990. An evaluation of platinum-DNA adduct levels relative to known prognostic variables in a cohort of ovarian cancer patients. Cancer Res. 50:2256-2260.

30. Parker, R. J., M. C. Poirier, F. Bostick-Bruton, J. Vionnet, V. A. Bohr, and E. Reed. 1991. The use of peripheral blood leukocytes as a surrogate marker for cisplatin drug resistance: studies of adduct levels and ERCC1. Brookhaven Symp. Biol. In press. 